\title{
Decision Making on Bus Splitting Locations Using a Modified Fault Current Constrained Optimal Power Flow (FCC-OPF)
}

\author{
Hwachang Song ${ }^{\dagger}$, Panagis N. Vovos*, Kang-Wook Cho** and Tae-Sun Kim**
}

\begin{abstract}
This paper presents a method of decision on where bus splitting is needed to reduce fault current level of power systems and to satisfy the fault current constraints. The method employs a modified fault current constrained optimal power flow (FCC-OPF) with X variables for the candidate locations of splitting and for decision making on whether to split or not, it adopts soft-discretization by augmenting inversed U-shaped penalty terms. Also, this paper discusses the procedure on the adequate selection of bus splitting locations based on the results of the modified FCC-OPF, to reduce the total number of the actions taken.
\end{abstract}

Keywords: Bus splitting, Decision making, Fault current constraints, Optimal power flow, Rerun optimization process

\section{Introduction}

This paper presents a method of decision making on adequate selection of bus splitting locations to reduce fault current level considering fault current constraints. When power systems experience the increase in load demand, maintaining transfer capability of the system needs be regarded as one of the main factors for secure system operation [1-6]. However, for those systems with heavily looped transmission networks after generation and transmission expansion, fault current levels might be severely increased and some can exceed the breaking capacity of the circuit breakers. If the circuit breaker at the location of interest has less breaking capacity than the fault current level, fault isolation cannot be guaranteed, hence the impact of the fault could propagate through the system. This paper focuses on countermeasures applicable to reduce the fault current level.

Several countermeasures, as in the literature [7-8], can be taken to properly deal with the increase in fault current level. Of them, the best technical solution is to simply equip new circuit breakers with higher short circuit capacity at critical locations, but this solution carries both monetary and time cost. In operational planning stages, available countermeasures are limited to bus splitting, line opening and opening network loops. Making decisions on whether to split the candidate buses to reduce fault current levels is the main target of this paper. This paper employs a modified fault current constrained optimal power flow (FCC-OPF). The concept of FCC-OPF was

$\dagger$ Corresponding Author: Dept. of Electrical and Information Engr. Seoul National University of Science \& Technology, Korea. (hcsong@seoultech.ac.kr)

* Dept. of Electrical and Computer Engr., University of Patras, Greece. (panagis@upatras.gr)

** Korea Power Exchange (KPX), Korea. (\{jokangu, ktsn\}@kpx.or.kr) Received: February 2, 2015; Accepted: October 9, 2015 proposed in [9-10]. In [9-10], the main goal was to evaluate network capacity considering the installed circuit breakers' ratings using sequential quadratic programming, on the assumption that the study system has some margins of fault currents to the limits. However, this paper mainly considers the base case system with excessive bus fault current levels and hence certain actions of topology modification need to be taken to reduce fault current levels in the operational planning stage.

A bus fault current can be formulated with a nonlinear function of the pre-fault voltage magnitude and the Thévenin equivalent impedance at the pre-defined fault location. In the problem of this paper, the equivalent impedance is an implicit function of the binary variables representing bus splitting actions. This paper, instead of direct incorporating the binary variables, introduces continuous reactances $(X \mathrm{~s})$ for the candidate bus splitting locations in the modified FCC-OPF. The formulation takes penalty functions to enforce the continuous $\mathrm{X}$ variable to the two discrete points, meaning whether to split or not. This paper explains how to determine the Jacobian and Hessian terms that need to be added by the introduction of the $\mathrm{X}$ variables. Because the modified FCC-OPF tackles the power flow problem and countermeasure determination for fault current reduction in one formulation, it can properly deal with the effect of nonlinearity by the network change on power flow solutions.

In addition, this paper discusses the overall procedure on the adequate selection of bus splitting locations based on the results of the modified FCC-OPF. This paper is a revised and extended version of a conference paper that appeared in [11]. It was noticed that there were several cases with the intermediate values of $X \mathrm{~s}$ even though the penalty terms for soft-discretization were imposed to the optimization formulation, and that the adequate selection of bus splitting locations critically affected the total 
number of the actions. In this paper, a decision making procedure with the modified FCC-OPF is described to effectively remove the infeasibility of the decision variables and to reduce the number of actions taken. This paper includes the results applying the method to a 28-bus test system and Korean power systems with 1,338 buses to illustrate its performance.

\section{Fault Current Constrained Optimal Power Flow (FCC-OPF)}

Optimal power flow (OPF) is to find optimal setting of the control vector, minimizing the objective function or maximizing the operational efficiency within the feasible region. One of typical OPF aims is to re-dispatch active power generation to minimize the total generation cost of the system. In the OPF formulation, it is assumed that one is informed of the followings: active and reactive power generation capabilities, sizes of loads, thermal limits of transmission lines, specifications of transformers and other equipments. The control vector, determined during the optimization process, may include active and reactive power generation output, tap ratios and/or phase shifts of transformers, settings of switchable shunt devices, and shedding amounts of interruptible loads. The state vector in the formulation needs to be defined, and it basically includes bus voltage magnitudes and angles for the whole buses, but for the system with new equipments, more variables can be incorporated into the state vector. Pertaining to the expected fault current, network topology and patterns of generation and load are important. As the load demand gets increased, the expansion of generation and transmission are needed to support a specified load demand. For those systems with the possibility of excessive fault current levels, they should be properly managed. In this sense, applying fault level constraints to OPF formulations were proposed [9]. It is noted that in [9] the constraints are based on line fault currents when a 3 phase short circuit fault is applied to one location of the system. However, in real applications, bus fault currents might be mainly considered in decision making of circuit breakers' (CB) rating in the planning or operational planning stage. In the Korean power system, 1.5 CB configurations are used for extra high voltage (EHV) substations. When a short-circuit fault applies near an EHV substation, two CBs need to be open to isolate the section experiencing the fault. In real situation, there might be a time delay between two CB openings, and the second $\mathrm{CB}$ to be opened needs to break the total fault current. Thus, it is quite reasonable to use bus fault currents in the decision making.

FCC-OPF is an OPF including fault current constraints. It can be said that fault current constraints (FCC) are one type of operational constraints to manage fault current security level. Within the physical limits imposed by the switchgear equipment at substations, the dispatch of control vectors of OPF can be made, similar to other operational constraints, assuming that the dispatch minimizing the objective function tends to violate the constraints.

Basically, fault current constraints are those applied to the magnitude of the short-circuit current. In this paper, the symmetrical component is only considered for the constraints. If one would like to take into account asymmetrical fault current, the $\mathrm{R} / \mathrm{X}$ ratio at each location also needs to be calculated and incorporated into the formulation of the constraints. As an approximate method for the decaying Direct Current component, a certain correction factor can be multiplied to the initial symmetrical component.

As mentioned above, if the system operators are concerned about the total bus fault current at each location because of the system property, then FCCs should be functioned of bus fault currents not line fault currents. The formulation of an FCC, used in this paper, is as follows:

$$
\frac{V_{f}}{\left|Z_{f, f}\right|} \leq I_{f, \text { max }}
$$

where $V_{f}$ stands for pre-fault voltage magnitude at the fault location, $Z_{f, f}$ represents the Thévenin impedance at the location.

For the calculation of the initial 3-phase short-circuit fault current, in conventional fault analysis, the bus admittance matrix needs to be constructed. For the construction of the matrix, branch impedances as well as tap ratios are needed and shunt admittances for loads, generators, and other shunt elements are added to the corresponding diagonal terms of the matrix. Conventionally, the loads are converted into admittances as follows:

$$
y_{L i}=\frac{P_{L i}-j Q_{L i}}{V_{L i}^{2}}
$$

where $P_{L i}$ and $Q_{L i}$ are active and reactive load at the i-th load bus, and $V_{L i}$ denotes the voltage magnitude at the i-th bus. Because of the added load admittance terms, the Thévenin impedance at the fault location can be affected by voltage magnitudes of the buses with loads. In [12], the impact of the change in load voltage magnitudes were investigated, and it is concluded that whether to consider the voltage magnitude change or not does not have a significant impact on the final solutions.

If the system fault current levels are manageable within the switchgear ratings, FCC-OPF can provide optimal operating points, for example, minimizing the generation costs and also satisfying the operational limits including FCCs. In addition, as in [10], it can provide the maximum installation capacity of distributed generators on distribution feeders, not violating FCCs. In the application to those power systems with the excess of fault current levels, fault current constraints cannot be satisfied without 
any taking remedial actions at several substations. Thus, a decision making is needed on which locations bus splitting is required. In [13], a conceptual framework for network reconfiguration was described, which was based on the fundamental formulation of FCC-OPF. In [14], an OPF with fault current constraints was applied for allocation of fault current limiters. In [15], a decentralized OPF including fault current constrained was proposed for generation system planning with a superconducting fault current limiter.

\section{Decision Procedure for Bus Splitting Locations with the Modified FCC-OPF}

\subsection{Modified FCC-OPF}

For those systems with excessive fault current levels, various types of countermeasures can be taken in the expansion and operational planning stage [7-8]. The best solution to the problem is simply to install new switchgears with adequate ratings, but an amount of investment is needed. Plus, the rating of commercial CBs seems to be limited, even though some conducted to develop new types of switchgears with higher ratings in the research stage. Alternatively, network owners might choose to insert series fault limiting reactors on branches in order to increase the equivalent impedance and hence to reduce expected fault current. This measure also has an important cost.

When the rating of the installed $\mathrm{CB}$ does not cover the fault current level, there are a few options that system operators can apply in the operational planning stage. The countermeasures of interest in operational planning are bus splitting and line opening, but this paper focuses on bus splitting. If the number of bus splitting is increased, the efficiency of system operation might be degraded, especially in contingent states. Thus, reducing the number of the actions would be desirable.

This paper adopts a modified version of FCC-OPF as the main solver in the decision making procedure. The procedure indeed determines whether to split buses for the predetermined candidate locations. As a preprocessing procedure, the candidate locations are decided and the network topology is modified so that the algorithm only focuses on the decision making on whether to split or not. In the problem, it is obvious that one of the decision variables is binary in the problem, but in this paper, a new continuous variable is introduced and it is the reactance $(X)$, inserted into the candidate location of bus splitting or

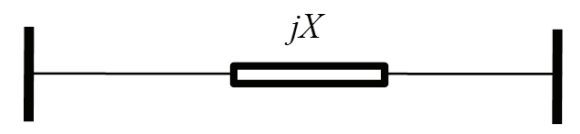

Fig. 1. Reactance inserted into a candidate location in the modified FCC-OPF line opening as shown in Fig. 1. As the reactance on is increased, the electrical distance between two buses for one candidate location is increased.

For the OPF problem with topology modification, it is desirable to use nonlinear interior point methods (NIPM) other than sequential quadratic programming (SQP). When not using Hessian terms as in the SQP formulation, the effect of the topology change by bus splitting actions on fault current constraints cannot be properly considered. The impact of the inserted reactance needs to be reflected into the formulation of the modified FCC-OPF, especially for the construction of the Jacobian and Hessian matrix. Most importantly, the increase of an inserted reactance may cause the increase in Thévenin impedances at some buses and then it further results in the reduction of fault current levels. Thus, this property should be considered in (1); in other words, in (1) $Z_{f, f}$ is not a constant value anymore and it should be a function of the inserted reactances. Then the formulation of an FCC needs to be modified as follows:

$$
\left|I_{f}\right| \equiv \frac{V_{f}}{\left|Z_{f, f}\left(X_{1}, X_{2}, . . X_{k}, X_{m}\right)\right|} \leq I_{f, \max }
$$

where $X_{k}$ represents the reactance, inserted into the $k$-th candidate remedial action location. It is assumed that there are $m$ continuous reactance variables.

When incorporating $X$ variables into the state vector in the optimization formulation, Jacobian and Hessian terms of network equality constraints and FCCs need to be provided for NIPM. It is not that difficult to formulate the Jacobian and Hessian terms for the network equality constraints, so this paper focuses on how to obtain those terms for the FCCs. Fig. 2 shows the illustration of the change of Thévenin impedance at the fault location by switching on the branch with $j X$ connecting bus $k$ and $l$.

By applying the direct $Z_{b u s}$ building algorithm as in [16], which was based on the Sherman-Morrison-Woodbury (S$\mathrm{M}-\mathrm{W})$ formula [17], the change in the diagonal element at $(f, f)$ in $Z_{b u s}$ with respect to $j X$ can be explained as follows:

$$
\underline{Z}_{f, f}=Z_{f, f}-\frac{\left(Z_{f, k}-Z_{f, l}\right)\left(Z_{k, f}-Z_{l, f}\right)}{Z_{k, k}-Z_{l, k}-Z_{k, l}+Z_{l, l}+j X}
$$

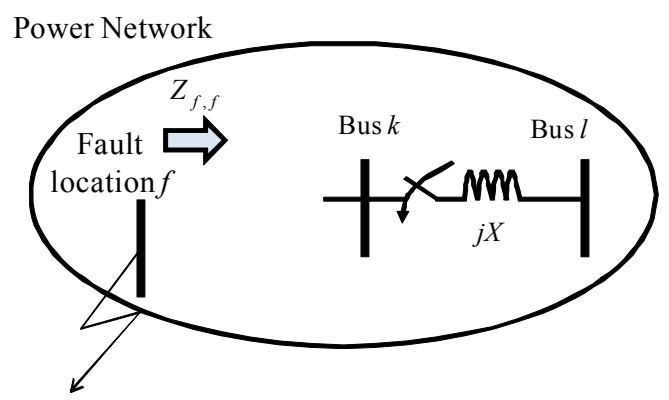

Fig. 2. Thévenin impedance at the fault location by switching on the branch with $\mathrm{j} X$ 
where $Z_{f, f}$ and $\underline{Z}_{f, f}$ denote the $(f, f)$ element before and after switching on the branch with $j X$. In right hand side of (4), the subscripts represent the locations of $Z_{b u s}$ before switching on $j X$.

To further evolve (4), complex number $B$ and $C$ are introduced as follows:

$$
\begin{gathered}
B \equiv\left(Z_{f, k}-Z_{f, l}\right)\left(Z_{k, f}-Z_{l, f}\right)=B^{x}+j B^{y} \\
C \equiv Z_{k, k}-Z_{l, k}-Z_{k, l}+Z_{l, l}+j X=C^{x}+j C^{y}
\end{gathered}
$$

where superscripts $x$ and $y$ represent real and imaginary part, respectively. One can notice that $C^{x}$ and $C^{y}$ are functions of $X$.

Substituting (5) and (6) for the numerator and denominator of the second term in the right hand side of (4) yields:

$$
\begin{aligned}
\underline{Z}_{f, f} & =Z_{f, f}^{x}+j Z_{f, f}^{y}-\frac{B^{x}+j B^{y}}{C^{x}+j C^{y}} \\
& =Z_{f, f}^{x}-\frac{B^{x} C^{x}+B^{y} C^{y}}{\left(C^{x}\right)^{2}+\left(C^{y}\right)^{2}}+j\left(Z_{f, f}^{x}-\frac{B^{x} C^{x}+B^{y} C^{y}}{\left(C^{x}\right)^{2}+\left(C^{y}\right)^{2}}\right) \\
& =D^{x}+j D^{y}
\end{aligned}
$$

where $D^{x}$ and $D^{y}$ are the real and imaginary part of $\underline{Z}_{f, f}$, respectively.

For FCC of (3), the magnitude of the Thévenin impedance is required, and it can be explained as follows:

$$
\left|\underline{Z}_{f, f}\right|=\sqrt{\left(D^{x}\right)^{2}+\left(D^{y}\right)^{2}}
$$

The first derivative of $\underline{Z}_{f, f}$ with respect to $\mathrm{X}$ can be determined as follows:

$$
\begin{aligned}
\frac{d\left|\underline{Z}_{f, f}\right|}{d X} & =\frac{d \sqrt{\left(D^{x}\right)^{2}+\left(D^{y}\right)^{2}}}{d X} \\
& =\frac{D^{x} \frac{d D^{x}}{d X}+D^{y} \frac{d D^{y}}{d X}}{\sqrt{\left(D^{x}\right)^{2}+\left(D^{y}\right)^{2}}}
\end{aligned}
$$

In (9), $d D^{x} / d X$ and $d D^{y} / d X$ can be obtained using their expressions as in (7). Then (9) can be evolved as follows:

$$
\begin{aligned}
& \frac{d\left|\underline{Z}_{f, f}\right|}{d X}=\frac{-D^{x} \frac{F B^{y}-2 E C^{y}}{F^{2}}+D^{y} \frac{F B^{x}-2 E C^{y}}{F^{2}}}{\sqrt{\left(D^{x}\right)^{2}+\left(D^{y}\right)^{2}}} \\
& E \equiv B^{x} C^{x}+B^{y} C^{y} \\
& F \equiv\left(C^{x}\right)^{2}+\left(C^{y}\right)^{2}
\end{aligned}
$$

Using (10), the first derivative of (3) with respect to $\mathrm{X}$ can be expressed as follows:

$$
\begin{aligned}
& \frac{d\left|I_{f}\right|}{d X}=\frac{d\left(\frac{V_{f}}{\left|\underline{Z}_{f, f}\right|}\right)}{d X}=-\frac{V_{f}}{\left|\underline{Z}_{f, f}\right|^{2}} \frac{d\left|\underline{Z}_{f, f}\right|}{d X} \\
& =-\frac{V_{f}}{\left|\underline{Z}_{f, f}\right|^{2}} \frac{-D^{x} \frac{F B^{y}-2 E C^{y}}{F^{2}}+D^{y} \frac{F B^{x}-2 E C^{y}}{F^{2}}}{\sqrt{\left(D^{x}\right)^{2}+\left(D^{y}\right)^{2}}}
\end{aligned}
$$

In the similar way, second derivatives of an FCC can be formulated for the Hessian components. For the purpose, Eq. (4) needs to be slightly modified so that Thévenin impedance before switching on two selected $X \mathrm{~s}$ is explicitly shown. This paper does not include the formulations of second derivatives of FCCs, $d^{2}\left|I_{f}\right| / d X_{1} d X_{2}$ and $d^{2}\left|I_{f}\right| / d V_{f} d X$, because of their long expression. Also, Jacobian and Hessian terms for power flow equations and flow constraints with respect to $X$ s are implemented on the modified FCC-OPF.

Since the main variables are continuous in (3), a soft discretization is needed during the solution procedure. If the inserted reactance is close to 0 or $X_{\min }$, the candidate location is considered as integrated; if it is large enough, close to $X_{\max }$, the location is regarded as opened. In this paper, $X_{\min }$ and $X_{\max }$ are set to 0.0001 and 10.0 [pu], respectively. Also a penalty term is added in FCC-OPF formulation, which is the reversed U-shaped objective function to enforce the continuous $X$ variables to the two discrete points, meaning whether to split or not, as shown in Fig. 3.

In Fig. 3, $K_{1}$ and $K_{2}$ are the factors for decision making of whether to integrate or split, respectively. When a reactance is less than and equal to $K_{1} X_{\min }$ at FCC-OPF solution, the location is considered as integrated; when it is greater than or equal to $K_{2} X_{\max }$, it is as open. The setting of $K_{1}$ and $K_{2}$ can affect the total iteration number of the solution procedure. Because $X_{\min }$ is usually set to a small value, the setting of $K_{1}$ might affect the total iteration number of the proposed algorithm more than that of $K_{2}$.

\subsection{Overall solution procedure}

Even though the penalty term for discretization is applied to the modified FCC-OPF, the solution from onetime run might contain some $X$ variables with intermediate values in the range of $\left(K_{1} X_{\min }, K_{2} X_{\max }\right)$. This is because some other operational constraints maybe binding. This phenomenon occurs frequently in real-sized system application. Then more iteration is needed for the final decision in a certain outer loop based on the results obtained from the modified FCC-OPF. Fig. 4 illustrates the overall flowchart of the actions in the decision making procedure. Using the proposed method, feasible solutions for all test systems has been obtained, under the conditions that enough resources of countermeasures were provided to satisfy the FCCs in the problem. 


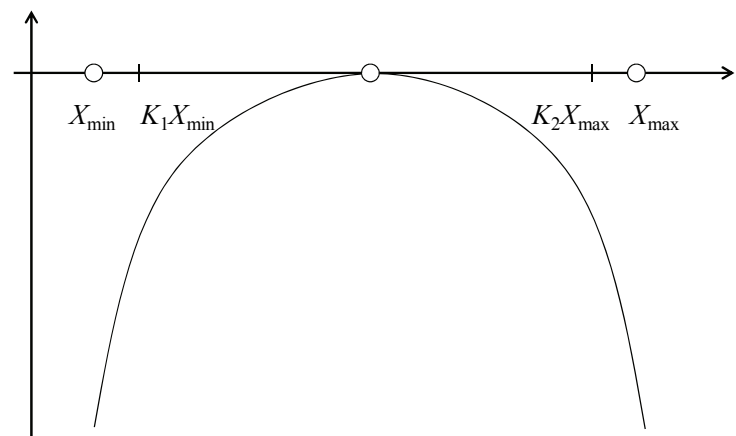

Fig. 3. Reversed U-shape penalty term for discretization

The whole procedure is explained in more detail. In step 1, the algorithm performs a conventional OPF with traditional operating constraints only; that is, the original network configuration is maintained. From the operating point, in step 2, fault analysis is conducted for those buses with FCCs for step 3, which is to check whether there are any FCC violations. If there are any violated FCCs, then the procedure for decision making on bus splitting with the modified FCC-OPF will be run; otherwise, the initial solution is accepted as the final one.

In step 4, effective $X$ variables are selected from the predefined set to reduce the number of decision variables. For the purpose, the amount of fault current reduction on the violated FCCs by each bus splitting candidate location is evaluated. When the amount of fault current reduction is more than the predefined threshold value the corresponding candidate gets into the group of effective splitting locations. In step 5, the modified FCC-OPF is performed, from the initial point obtained in step 2 . In the procedure of modified FCC-OPF, all the constraints including critical FCCs and the reversed U-shape penalty terms for $X \mathrm{~s}$ are considered. When all the $X \mathrm{~s}$ at the solution from FCCOPF are within the decision making region with $K_{1}$ and $K_{2}$ in the Fig. 2, the solution is accepted as final and then the procedure goes to step 9 for output analysis. For the 1st iteration solution of most cases, however, some $X$ s have intermediate values, not within the decision making region, because of some binding operational constraints.

In step 7, the algorithm further assesses the solution information, especially the value of each $X$, to select most effective $X$ variables from those with intermediate values and to force them to $X_{\max }$ in step 8 . The locations with largest intermediate values are considered as most effective among them. Also Lagrangian multipliers of binding fault current constraints can be used to indicate most critical CBs. To speed up the solution process for large power systems, this paper adopts a parallel selection method. The selection method determines the affected bus group by each bus splitting from the location with the highest intermediate value. If the affected bus group by the examined bus splitting does not overlap that by the

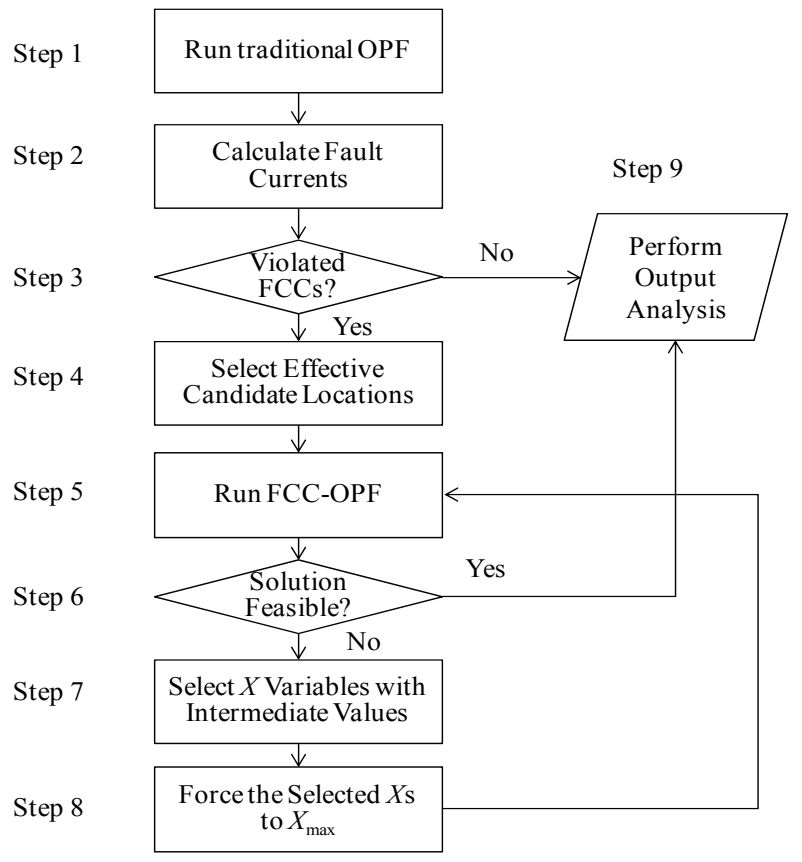

Fig. 4. Overall decision making procedure with the modified FCC-OPF

previously selected splitting locations. The other $X$ s with intermediate values are set to $X_{\min }$ for the next run of the modified FCC-OPF. By setting them to $X_{\max }$, its impact on fault current reduction is increased. Thus, one can expect that the other $X \mathrm{~s}$ with intermediate values have more chance to be close to $X_{\min }$.

Then the algorithm goes back to step 5, rerunning the modified FCC-OPF with the setting of $X \mathrm{~s}$ as a new starting point. The results of rerunning the algorithm might be quite different, depending on cases. After rerunning FCC-OPF, some selected Xs stay at the position and others move back to intermediate values. However, the decision making on whether to split for each candidate location was all accomplished in the final rerun iteration for most of cases. If action resources on candidate locations can cover the required reduction in fault currents at critical buses, then the algorithm can provide feasible settings for the decision making.

The proposed method based on the modified FCC-OPF was implemented in MATLAB. As the OPF platform, MATPOWER has been chosen because it provides an extensible OPF structure. The structure allowed the authors to augment the formulation of the additional constraints (FCCs) and the user defined objective functions without recoding the additional portions that are shared with the standard OPF formulation [18]. It is noted that for the large-scaled system simulation, NIPM in KNITRO [19] was chosen as the external solver. For the modified FCC$\mathrm{OPF}$, the information on first and second derivatives, required by the inclusion of Xs and FCC of (3), were provided to the external solver. 


\section{Numerical Results}

\subsection{8-bus test system}

The algorithm of this paper was tested on a 28-bus test system, which was a modified version of the sample system in PSS/E application guide [20]. As in Fig. 5, five buses $(3,4,7,18,21)$ were split into two portions. Each portion was connected with the other through a switch. Also, it was assumed that buses 1 and 2 were connected with a switch. Thus, there are 6 candidate locations for bussplitting. Other countermeasures could be taken such as opening of transmission lines, but this example focuses on bus-splitting for the simplicity of illustration.

The primary objective function was the minimization of production cost for FCC-OPF. The coefficients for the generators' quadratic functions are shown in Table 1. The resistance and reactance for each generator for fault current analysis are given in Table 2 . In the example, 6 cases with different sets of fault current constraints were simulated. In Table 3, the limits of FCCs in each case are shown. $X_{\min }$ and $X_{\max }$ are set to 0.0001 and 10.0 , respectively, and $\mathrm{K}_{1}$

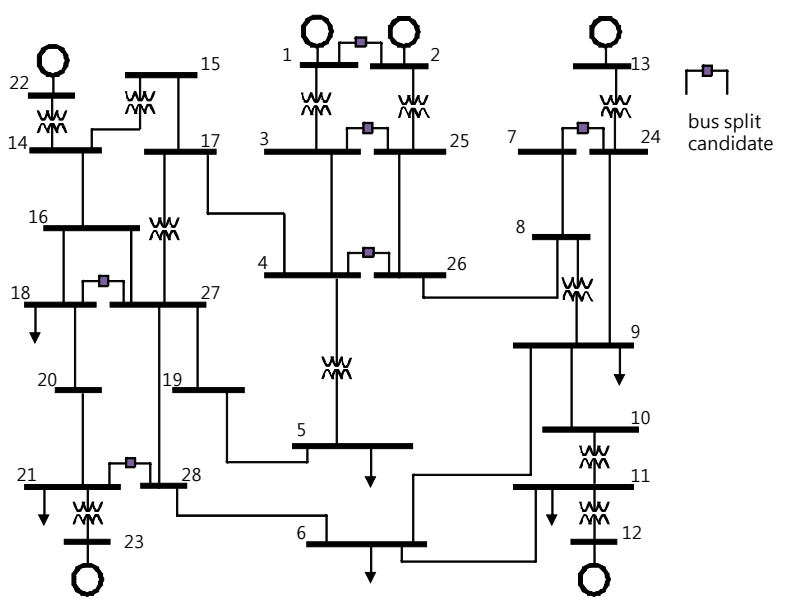

Fig. 5. One-line diagram of 28-bus test system

Table 1. Generators' quadratic cost function coefficients

\begin{tabular}{c|c|c|c}
\hline Bus & Quadratic term & Linear term & Constant term \\
\hline 1 & 0.000 & 10.000 & 0.000 \\
\hline 2 & 0.000 & 10.000 & 0.000 \\
\hline 12 & 0.010 & 12.000 & 0.000 \\
\hline 13 & 0.010 & 10.833 & 0.000 \\
\hline 22 & 0.008 & 12.000 & 0.000 \\
\hline 23 & 0.007 & 11.669 & 0.000 \\
\hline
\end{tabular}

Table 2. Generators' impedances with machine base

\begin{tabular}{c|c|c|c}
\hline Bus & Resistance $(\mathrm{pu})$ & Reactance $(\mathrm{pu})$ & $\mathrm{S}_{\text {mach }}[\mathrm{MVA}]$ \\
\hline 1 & 0.010 & 0.6 & 900 \\
\hline 2 & 0.010 & 0.6 & 900 \\
\hline 12 & 0.010 & 0.5 & 1000 \\
\hline 13 & 0.010 & 0.4 & 725 \\
\hline 22 & 0.010 & 0.7 & 1000 \\
\hline 23 & 0.010 & 0.7 & 130 \\
\hline
\end{tabular}

Table 3. Fault current constraints for each case

\begin{tabular}{c|c|c|c}
\hline Case & $\begin{array}{c}\text { Limits of FCC } \\
\left.\text { (bus \#, } I_{f, \max }[\mathrm{pu}]\right)\end{array}$ & Case & $\begin{array}{c}\text { Limits of FCC (bus \#, } \\
\left.I_{f, \max }[\mathrm{pu}]\right)\end{array}$ \\
\hline 1 & $(1,45)$ & 1 & $(1,45)$ \\
\hline 2 & $(4,40)$ & 2 & $(4,40)$ \\
\hline 3 & $(1,45)$ & 3 & $(1,45)$ \\
\hline 4 & $(1,45)$ & 4 & $(1,45)$ \\
\hline 5 & $(1,45)$ & 5 & $(1,45)$ \\
\hline 6 & $(19,32)$ & 6 & $(19,32)$ \\
\hline
\end{tabular}

Table 4. $\mathrm{X}$ variables after the 1 st and 2 nd run

\begin{tabular}{c|c|c}
\hline Candidate & $X$ after 1st run & $X$ after 2nd run \\
\hline $7-24$ & 0.0001 & 0.0001 \\
\hline $3-25$ & 0.0076 & 0.0001 \\
\hline $4-26$ & 0.0001 & 0.0001 \\
\hline $18-27$ & 0.0001 & 0.0001 \\
\hline $21-28$ & 0.0001 & 0.0001 \\
\hline $1-2$ & 0.0304 & 9.9999 \\
\hline \hline Solution time $(\mathrm{s})$ & 0.54 & 0.30 \\
\hline
\end{tabular}

Table 5. Results of the algorithm for 6 Cases

\begin{tabular}{c|c|c|c|c|c|c}
\hline \multirow{2}{*}{ Candidate } & \multicolumn{7}{|c}{ Case } \\
\cline { 2 - 7 } & 1 & 2 & 3 & 4 & 5 & 6 \\
\hline $7-24$ & 0.0001 & 0.0001 & 0.0001 & 0.0001 & 0.0001 & 0.0001 \\
\hline $3-25$ & 0.0001 & 0.0001 & 0.0001 & 0.0001 & 0.0001 & 0.0001 \\
\hline $4-26$ & 0.0001 & 9.9999 & 9.9999 & 9.9999 & 9.9999 & 9.9999 \\
\hline $18-27$ & 0.0001 & 0.0001 & 0.0001 & 0.0001 & 0.0001 & 0.0001 \\
\hline $21-28$ & 0.0001 & 0.0001 & 0.0001 & 9.9999 & 9.9999 & 0.0001 \\
\hline $1-2$ & 9.9999 & 0.0001 & 9.9999 & 9.9999 & 9.9999 & 0.0001 \\
\hline $\begin{array}{c}\text { Solution } \\
\text { time (s) }\end{array}$ & 0.84 & 0.52 & 0.87 & 0.89 & 2.05 & 0.63 \\
\hline Total runs & 2 & 2 & 3 & 3 & 4 & 2 \\
\hline
\end{tabular}

and $\mathrm{K}_{2}$ are set to 1.1 and 0.9 in Fig. 2 .

As described in section 3 , a reactance is inserted into each candidate location, and it is regarded as a main variable in the decision making. The number of candidate locations is small in this example, so step 4 was skipped. For Case 1, in Table 3, the X variables after the 1 st and 2 nd run of FCC-OPF are shown in Table 4. After the 1st run of FCC-OPF, two locations (3-25, 1-2) had intermediate values, so step 7 and step 8 in Fig. 3 were performed, selecting effective $\mathrm{X}$ variables and forcing them to $X_{\max }$. In this example, one $X$ variable with the highest intermediate value was chosen and it was (1-2). Then FCC-OPF was redone. As described in Table 4, $\mathrm{X}$ variables after the 2 nd run are in the decision region. With this result, one can notice that the candidate location of (1-2) needs to be split for the FCC in Case 1. At the solution of the 1st run, the shadow cost of the FCC was 375250 , and at that of the 2 nd run, it was 0.0011 . Therefore, the FCC was successfully alleviated by the bus splitting.

Table 5 shows the results of the algorithm for 6 Cases, briefly. From Table 5, it can be known that to alleviate the FCC for Cases 4 and 5, 3 candidate locations, (4-26, 21-28, 1-2), need to be split, and that other cases require less than 3 split locations. In terms of the total runs of FCC-OPF, 
Table 6. Fault current levels after applying bus splitting for Case 5

\begin{tabular}{c|c|c|c}
\hline \multirow{2}{*}{ Buses } & \multicolumn{2}{|c|}{ Fault current [pu] } & \multirow{2}{*}{ Limits [pu] } \\
\cline { 2 - 3 } & Before splitting & After splitting & \\
\hline 1 & 55.8568 & 43.3295 & 45 \\
\hline 4 & 48.7682 & 37.6307 & 40 \\
\hline 19 & 35.7763 & 31.6701 & 32 \\
\hline 26 & 48.7418 & 32.644 & 40 \\
\hline 28 & 35.7065 & 30.179 & 33 \\
\hline
\end{tabular}

one can notice that the more splitting required, the more iteration needed to get the final decision. For Case 4, the 3rd run of FCC-OPF directly proposed two locations to be split, because the corresponding $X \mathrm{~s}$ are very close to $X_{\max }$. Thus, the total number of FCC-OPF runs for Case 4 was 3. Table 6 illustrates fault current levels of buses with FCCs for Case 5 before and after splitting. From Table 6, one can notice that all the FCCs are satisfied after the determined set of splitting.

\subsection{Korea 1338-bus system}

In this subsection, the test results of the algorithm with Korea 1338-bus system is described. The total load is $74,576[\mathrm{MW}]$ and the total generation is 75,720 [MW]. The number of generators is 319 and that of branches is 2,628. Without applying any countermeasures in the operational planning stage such as bus splitting and line opening, the fault current levels are quite severe. The transmission system operator takes action based on experience, in order to reduce fault levels. Thus decision making on whether to split buses on each candidate location using a more systematic method is desirable. In this simulation, $X_{\min }$ and $X_{\max }$ are set to 0.0001 and 10.0, respectively, and $\mathrm{K}_{1}$ and $\mathrm{K}_{2}$ are set to 10 and 0.9 , respectively.

The primary objective function is to minimize the deviation of generation from its initial pattern. As the basic test to the system, the conventional OPF, without FCCs and splitting candidates, was first performed. It solved the problem in 63 iterations and the maximum deviation is 9.9 [MW]. Then 14 bus-splitting candidates were added to the problem, but no FCCs were given. In the case, it took 34 iterations for convergence and the maximum deviation is 9.9 [MW], the same as the previous case. The decision was that no bus-splitting was required. The number of splitting candidates is increased further to 64 . For convergence, it took 241 FCC-OPF iterations, and 5 more runs of FCC$\mathrm{OPF}$ are needed to clear $X \mathrm{~s}$ with intermediate values. The maximum deviation at the solution was 0.4 [MW], lower than those of the previous cases. From the fundamental tests run on several simulated test and large-scale systems, it was concluded that the algorithm with continuous $X$ variables, inserted into the candidate locations, could provide adequate solutions.

The case with 1 FCC and 34 splitting candidates was tested. The internal bus number for the FCC was 204 and

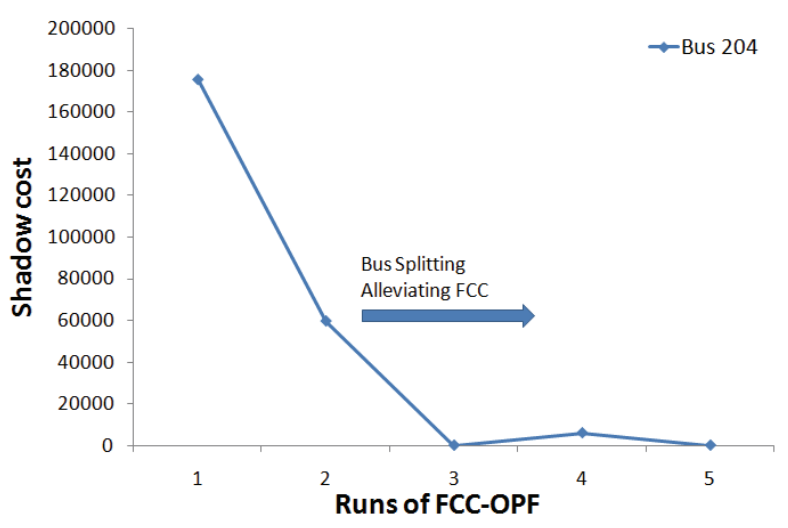

Fig. 6. Shadow cost related to the FCC at bus 204

its maximum fault current limit was 239 [pu]. As in Fig. 3, the conventional OPF was first solved. By fault analysis for the OPF solution, it was found that the FCC violates the limit; the base fault current of the bus was 510 [pu], and that some actions need to be taken to get the fault current on the location reduced within the maximum limit. Then, FCC-OPF was solved with 1 FCC and the 34 splitting candidates. It was solved done with one splitting candidate being open and several candidates having intermediate values; that is, the solution was not feasible yet. In this case, only one FCC is considered so that the algorithm brings splitting candidates to their maximum values one by one. However, in cases where several FCCs need to be satisfied, effective candidates are forced to $X_{\max }$. The procedure from steps 5 to 8 were repeatedly done, until the solution proved to be feasible. To get the feasible solution it took 5 runs of FCC-OPF, and the simulation time was $1.3[\mathrm{~h}]$ using the computing system with Intel QuadCPU 6600 with $2.4 \mathrm{GHz}$. The maximum deviation from the original dispatch is 0.4 [MW]. As result, 5 candidates are determined to be split and the fault current at bus 204 at the final solution was 238 [pu]. Fig. 6 shows the change of shadow cost related to the applied FCC.

Next, the case with 3 FCCs and 49 splitting candidates was examined, and for the case two simulation scenarios were performed. Table 7 shows the list of the candidate locations. The first scenario is to simulate the algorithm with series selection in step 7 and 8 . That is, at each iteration one candidate location was chosen from those with intermediate $X$ values after the modified FCC-OPF and the selected $X$ was forced to the maximum value. The other scenario is to simulate the algorithm with parallel selection using the information of the splitting candidate set. The parallel selection is based on the affected bus group obtained by each bus splitting in the order of high intermediate $X$ values. If the affected bus group by a splitting candidate with a intermediate $X$ value is different from those by previously examined splitting, the selection of the candidate might be reasonable.

Fig. 7 shows the change of the $X$ variables during the rerun process of FLC-OPF with applying the method of 
Table 7. List of the 49 candidate locations

\begin{tabular}{c|c|c||c|c|c||c|c|c}
\hline Loc. & \multicolumn{2}{|c|}{ From-To } & Loc. & \multicolumn{2}{c|}{ From-To } & Loc. & \multicolumn{2}{c}{ From-To } \\
\hline \hline$\# 1$ & 41 & 42 & $\# 18$ & 205 & 206 & $\# 35$ & 494 & 495 \\
\hline$\# 2$ & 90 & 91 & $\# 19$ & 217 & 218 & $\# 36$ & 582 & 583 \\
\hline$\# 3$ & 100 & 101 & $\# 20$ & 264 & 266 & $\# 37$ & 536 & 537 \\
\hline$\# 4$ & 105 & 106 & $\# 21$ & 267 & 268 & $\# 38$ & 614 & 615 \\
\hline$\# 5$ & 107 & 108 & $\# 22$ & 273 & 274 & $\# 39$ & 620 & 621 \\
\hline$\# 6$ & 110 & 111 & $\# 23$ & 22 & 23 & $\# 40$ & 413 & 414 \\
\hline$\# 7$ & 128 & 129 & $\# 24$ & 31 & 32 & $\# 41$ & 784 & 785 \\
\hline$\# 8$ & 144 & 145 & $\# 25$ & 33 & 34 & $\# 42$ & 823 & 824 \\
\hline$\# 9$ & 131 & 132 & $\# 26$ & 62 & 63 & $\# 43$ & 825 & 826 \\
\hline$\# 10$ & 193 & 194 & $\# 27$ & 362 & 363 & $\# 44$ & 864 & 865 \\
\hline$\# 11$ & 203 & 204 & $\# 28$ & 249 & 250 & $\# 45$ & 838 & 839 \\
\hline$\# 12$ & 214 & 215 & $\# 29$ & 297 & 298 & $\# 46$ & 815 & 816 \\
\hline$\# 13$ & 223 & 224 & $\# 30$ & 313 & 314 & $\# 47$ & 938 & 939 \\
\hline$\# 14$ & 239 & 240 & $\# 31$ & 321 & 322 & $\# 48$ & 920 & 921 \\
\hline$\# 15$ & 248 & 241 & $\# 32$ & 325 & 326 & $\# 49$ & 900 & 901 \\
\hline$\# 16$ & 257 & 258 & $\# 33$ & 351 & 352 & \multicolumn{4}{|}{} \\
\hline$\# 17$ & 275 & 276 & $\# 34$ & 450 & 451 & & & \\
\hline
\end{tabular}



Fig. 7. Change of $X$ values when applying the rerun process with series selection

series candidate selection. At the first iteration of the rerun process, eight $X \mathrm{~s}$ have intermediate values, and then at each run one $X$ was chosen and set to the maximum value. From Fig. 7, it is noticed that the number of the infeasible $X \mathrm{~s}$ was reduced as the iteration increased and that at the final run the decision on the whole splitting candidates was made. In the first scenario, the algorithm needs to perform six runs of FCC-OPF. Fig. 8 illustrates the change of the $\mathrm{X}$ variables when applying the rerun process of FLC-OPF with parallel selection. Different from the results of the first scenario, it took four iterations of the rerun process and the set of the candidates to be open was the same as the first scenario. From this comparison, one can conclude that the rerun process with the parallel selection speeds up the decision process.

Lastly, the case with 127 FCCs and 57 splitting candidates was considered for the decision making simulation. For the feasible solution, 5 runs of the rerun process were needed and it took around 2.8589 [day], At the solution, 27 candidates were decided to be opened, satisfying the whole 127 FCCs. There were 12 active fault current constraints. Fig. 9 illustrates the fault currents of

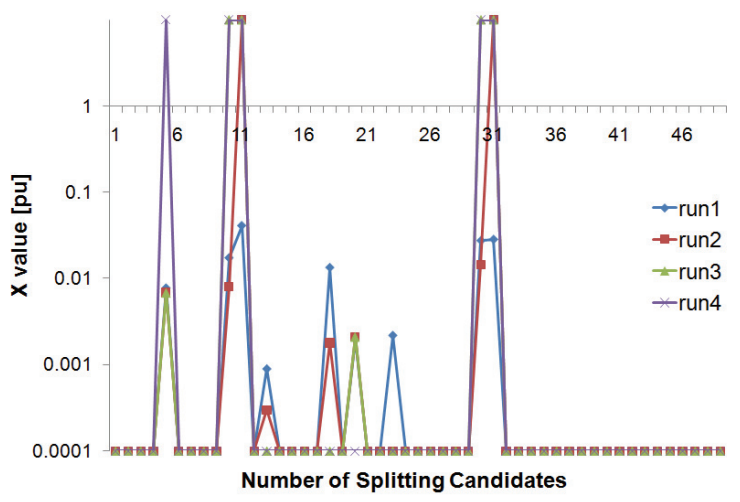

Fig. 8. Change of $X$ values when applying the rerun process with parallel selection

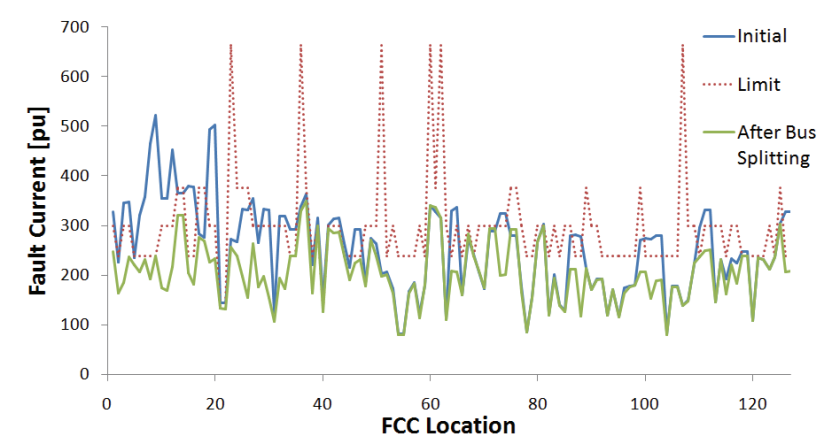

Fig. 9. Fault currents for the FCC locations with the limits

Table 8. Binding FCCs with shadow costs at the solution

\begin{tabular}{c|c|c|c|c}
\hline Bus \# & Base [pu] & After split [pu] & FCC [pu] & Shadow cost \\
\hline \hline 203 & 522 & 238 & 239 & 37 \\
\hline 217 & 145 & 133 & 133 & 94 \\
\hline 218 & 144 & 132 & 133 & 107 \\
\hline 50 & 293 & 239 & 239 & 613 \\
\hline 51 & 293 & 239 & 239 & 3.522 \\
\hline 340 & 316 & 299 & 299 & 25.261 \\
\hline 350 & 264 & 239 & 239 & 9.007 \\
\hline 552 & 243 & 239 & 239 & 4.058 \\
\hline 680 & 303 & 299 & 299 & 1.455 \\
\hline 837 & 295 & 237 & 239 & 15 \\
\hline 707 & 247 & 239 & 239 & 2.456 \\
\hline 708 & 247 & 239 & 239 & 1.939 \\
\hline
\end{tabular}

the FCC locations before and after the proposed algorithm with the relevant limits. Table 8 lists the locations of the 12 binding FCCs with fault currents before and after the decision making algorithm. The information of shadow costs might be used further to select critical CB locations. That is, it would be better to conclude that activate the CBs with high shadow costs are critical in terms of fault current.

The proposed algorithm for reducing fault current level through the system was based on the modified FCC-OPF with $\mathrm{X}$ variables added for the candidate splitting locations. Not directly dealing with the decision variables of whether to split on candidates might enhance the convergence rate. Also the allowance of active power dispatch may help the 
algorithm converge. Even though the reversed U-shape penalty terms for discretization are considered, the modified FCC-OPF cannot provide the feasible solution from the viewpoint of decision making on whether to open. Thus this paper implements the decision making algorithm with the concept of rerun optimizer, and it could provide solutions for most cases if the number of splitting candidates are enough to satisfy the given FCCs.

\section{Conclusion}

This paper describes a decision making algorithm for bus-splitting using a modified fault current constrained optimal power flow (FCC-OPF). The algorithm is to make decisions on where bus splitting needs to be applied for reducing fault current level of power systems within the $\mathrm{CB}$ ratings. For the purpose, the soft-discretization technique using continuous $\mathrm{X}$ variables was adopted in the formulation of FCC-OPF. However, some $\mathrm{X}$ variables converge to intermediate variables at the solution of FCC$\mathrm{OPF}$, so the further iterative procedure is needed to obtain feasible discrete solutions. Thus, this paper also presents simulation results with a rerun process with modified FCCOPF to effectively remove the infeasibility of the decision variables and to reduce the number of remedial actions taken. If enough number of splitting candidates are given which can cover the required reduction in fault currents for the given set of FCCs, then the algorithm could provide feasible sets of bus splitting. The future work in this subject obviously is how to speed up the solution process, possibly by the parallel computing as well as the approximated formulation on FCCs.

\section{Acknowledgements}

This work was supported by the research fund of Korea Power Exchange (KPX) and by the Human Resources Development of the Korea Institute of Energy Technology Evaluation and Planning (KETEP) grant funded by the Korea government Ministry of Trade, Industry \& Energy (No. 20154030200720).

\section{References}

[1] P. Kundur, Power System Stability and Control, McGraw-Hill, 1994.

[2] NERC, Available Transfer Capability Definitions and Determination, NERC Brochure, 1996.

[3] T. Van Cutsem and C. Vournas, Voltage Stability of Electric Power Systems, Springer, 1998.

[4] V. Ajjarapu, Computational Techniques for Voltage Stability Assessment and Control, Springer, 2006.
[5] H. Song, S. Kim, B. Lee, S. H. Kwon, and V. Ajjarapu, "Determination of interface flow margin using the modified continuation power flow in voltage stability analysis," IEE Proceedings-Generation Transmission and Distribution, vol. 148, Mar. 2001, pp. 128-132.

[6] B. Lee, H. Song, S.-H. Kwon, G. Jang, J.-H. Kim and V. jjarapu, "A study on determination of interface flow limits in the KEPCO system using modified continuation power flow (MCPF)," IEEE Trans. on Power Systems, vol. 17, Aug. 2002, pp. 107-125.

[7] N. Tleis, Power Systems Modelling and Fault Analysis: Theory and Practice, Newnes, 2008.

[8] G. Andersson, Modelling and Analysis of Electric Power Systems, ETH Zurich, 2008.

[9] P. Vovos and G. Harrison, "Optimal Power Flow as a Tool for Fault Level Constrained Network Capacity Analysis," IEEE Trans. on Power Systems, vol. 20, May 2005, pp. 734-741.

[10] P. Vovos and J. Bialek, "Direct Incorporation of Fault Level Constraints in Optimal Power Flow as a Tool for Network Capacity Analysis," IEEE Trans. on Power Systems, vol. 20, Nov. 2005, pp. 2125-2134.

[11] H. Song, P. Vovos, T.-S. Kim, K.-W. Cho, "Decision Making of Bus Splitting for Reduction of Fault Current Level Using a Novel Network Reconfiguration Algorithm with Rerun Optimizer," Proc. of 2014 CIGRÉ Session, Paris, France, 2014.

[12] H. Song, M. Y. Del Castillo, Jr, P. Vovos, T.-S. Kim, K.-W. Cho, "Varying Load Voltage Magnitude Impacts on Fault Level Constrained Optimal Power Flow," International Journal of Research in Engineering and Science, vol. 2, no. 2, 2014, pp. 39-43.

[13] P. Vovos, H. Song, K.-W. Cho, and T.-S. Kim, "A Network Reconfiguration Algorithm for the Reduction of Expected Fault Currents within Limits," Proc. of IEEE PES GM 2013, Vancouver, 2013.

[14] A. Khazali, M. Kalantar, "Optimal Power Flow Considering Fault Current Level Constraints and Fault Current Limiters," Int. J. of Electrical Power \& Energy Systems, vol. 59, July 2014, pp. 204-213.

[15] G.-H. Moon, Y.-M. Wi, K. Lee, and S.-K. Joo, "Fault Current Constrained Decentralized Optimal Power Flow Incorporating Superconducting Fault Current Limits (SFCL)," IEEE Trans. Applied Superconductivity, vol. 21, June 2011, pp. 2157-5160.

[16] P. M. Anderson, Analysis of Faulted Power Systems, Wiley, 1995.

[17] J. Sherman and W. J. Morrison, "Adjustment of an Inverse Matrix Corresponding to Changes in the Elements of a Given Column or a Give Row of the Original Matrix," Ann. Math. Statist., vol. 20, pp. 621, 1949.

[18] R. D. Zimmerman, C.E. Murillo-Sanchez, and R.J. Thomas, "MATPOWER: Steady State Operations, Planning, and Analysis Tools for Power Systems 
Research and Education," IEEE Trans. on Power Systems, vol. 26 Feb. 2011, pp. 12-19.

[19] Knitro Documentation Release 8.0, Ziena Optimization LLC, 2011.

[20] Power Technologies International, PSS/E 31.0 Users Guide, Siemens, 2007.

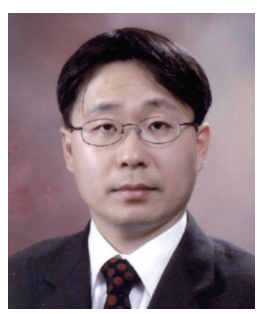

Hwachang Song received B.S., M.S. and $\mathrm{Ph} . \mathrm{D}$. in Electrical Engineering from Korea University in 1997, 1999 and 2003, respectively. He was a Postdoctoral Visiting Scholar at Iowa State University from 2003 to 2004, and a Post-doctor at Korea University from Sept. 2004 to March 2005. He was working as an Assistant Professor in the School of Electronic and Information Engineering, Kunsan National University, from 2005 to 2008. Currently, he is an Associate Professor in the Department of Electrical and Information Engineering, Seoul National University of Science \& Technology. His research interests include power system stability \& control, FACTS equipments, optimization applications, energy storage applications, and renewable energy

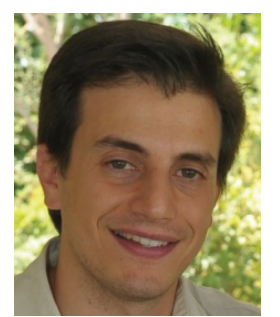

Panagis N. Vovos was born in Athens, Greece, on October 23, 1978. He received the MEng degree from the Electrical Engineering Department, University of Patras, Patras, Greece, in 2002. He received a scholarship from the University of Edinburgh, UK, where he completed his Ph.D. thesis in 2005. He completed his postdoctoral research in the new field of economic use of power system equipment in 2009, funded by the Hellenic State Scholarships Foundation. He is currently a Lecturer in University of Patras, Patras, Greece. His special fields of interest include efficient capacity allocation, equipment wear optimization, enhanced $\mathrm{OPF}$ and smart-grid power converters.

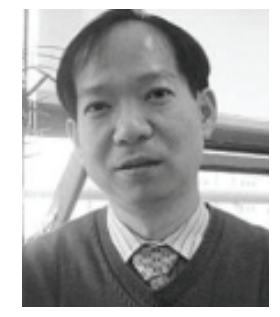

Kang-Wook Cho received his B.S. and M.S. degrees in Electrical Engineering from Seoul National University, Seoul, Republic of Korea, in 1986 and 1988 respectively. He is currently a team leader in Dept. of Market Development of KPX. His research interests include power system planning and operations such as regional load forecasting, optimization of generation and transmission investment, power system probabilistic assessment, and electricity market design

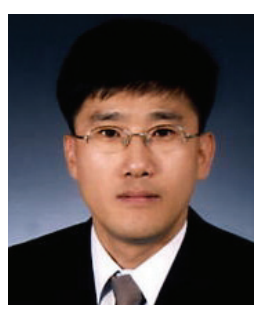

Tae-Sun Kim received his B.S. in Electrical Engineering from Dankook University in 1990. He worked for KEPCO from 1990 to 2001. He is currently a team leader in National Electric Power Control Center of KPX. 